\title{
The Improvement of Education Management for "Power Load Forecasting Technology" Curriculum
}

\author{
Yong-li Wang ${ }^{1}$, Li-sha Fan ${ }^{2}$, Dong-xiao Niu ${ }^{1}$ \\ 1) School of Economics and Management, North China Electric Power University,102206, Beijing, China \\ ${ }^{2)}$ Jibei Electric Power Company Limited Training Center, Beijing, 102488, China
}

\begin{abstract}
Electric power load forecasting course is an important course to power universities and power related school, and it has many quite different features from other courses. The paper analyzes some existing problems in this course, for example, a lot of mathematical knowledge is involved, the course itself is boring, there is a lack of practice, and assessment method is relatively single. After analyzing these problems, this paper put forward several measures, such as strengthening the interaction in class, organizing practice and reforming examination methods to provide references for the course reform in college.
\end{abstract}

Keywords — power load forecasting, educational reform, learning initiative

\section{“电力负荷预测技术”课程教育管理改进研究}

\author{
王永利 $^{1} \quad$ 樊立沙 $^{2} \quad$ 牛东晓 $^{1}$ \\ 1) 华北电力大学经济与管理学院, 北京, 中国 \\ 2) 国网冀北电力有限公司管理培训中心, 北京, 中国
}

\begin{abstract}
摘 要 电力负荷预测课程是电力院校以及开设电力相关专业的学校较为重要的课程, 其自身拥有很多与其他课程不同的特点。 本文通过分析当前电力负荷预测课程所存在的一些问题, 比如利用数学知识较多, 课程枯燥、缺少实践环节、考核方式较为单一等等, 进而提出加强课堂互动、组织实践环节以及改革考核方法等几项措施, 希望能对高校这门课程的改革提供借鉴意义。
\end{abstract}

关键词 电力负荷预测; 教育改革; 学习积极性

\section{1. 引言}

\section{1 课程简介}

“电力负荷预测技术及其应用”是电力院校以及其他高 校开设电力相关专业课程的专业主干课 ${ }^{1}$ 。负荷预测就是在 正确的理论原理指导下, 从已知的经济、社会发展和电力 需求情况出发, 通过对历史数据的分析与研究, 利用一套 能系统处理过去与未来负荷变化的可靠方法和手段, 对未

\footnotetext{
1 This research has been supported by Natural Science Foundation of China (71101048); It also has been supported by the Doctor spot special Foundation of NCEPU(20110036120001), supported by "Beijing philosophy and social science planning projects(13JGC098)", "the Fundamental Research Funds for the Central Universities".
}

来用电需求的发展趋势做出预先科学合理的估计和推测。 课程内容包括了电力负荷的相关基本概念、电力负荷的经 验技术与经典技术、趋势外推预测技术、回归模型预测技 术、随机时间序列预测技术、灰色预测技术等各种预测的 算法, 以及介绍了预测技术的新发展和负荷预测系列软件 等等。

\section{2 课程的重要性}

电力工业是国家非常重要的基础行业之一, 对于我国 的经济建设、国家安全、社会稳定、生活质量具有至关重 要的作用。随着电力工业的迅速发展以及我国电力市场改 革的不断推进, 对电力负荷预测技术的要求越来越高, 亟 待需要更多这方面的知识提高和人才培养。

电力负荷预测是电力系统调度、用电、计划、规划等 
管理部门的重要工作之一。提高负荷预测技术水平, 有利 于计划用电管理、合理安排电网运行方式和机组检修计划、 节约能源、降低发电成本、制定合理的电源建设规划以及 提高电力系统的经济效益和社会效益。随着我国电力事业 的发展, 电网的管理日趋现代化, 电力系统负荷预测问题 的研究也逐渐成为实现电力系统管理现代化的重要内容。 由于高校是培养人才的发源地, 所以, 在高校内开展的相 关课程的教育至关重要。

\section{2. 相关知识储备分析}

\section{1 前期准备}

“电力负荷预测技术及其应用” 的课程与数学知识有着 不可分割的关系。在很多的预测算法中, 不仅用到了基本 的数学模型, 比如弹性系数、指数平滑法, 另外还有在回 归预测中运用到概率论与数理统计的知识, 在灰色预测中 运用到线性代数的知识等等。所以, 在学习电力负荷课程 之前, 应该对数学知识进行一个有效的整理与复习, 让同 学们可以在学习负荷预测的算法中不会因为数学知识的遗 忘或者不贯通而止步不前。另外, 对于专业的电力相关词 汇, 也要提前做一定的了解与解释, 这样可以使学生学习 理解起来更加轻松。

\section{2 学习过程中}

学习过程中, 要使每一位同学认真去理解每一种预测 算法的思想, 不要硬记公式, 将每一种方法讲解透彻, 可 以让学生自己从头推导一遍。并使用电力负荷预测的软件 进行操作, 加深理解。另外, 建议学生对新学到的相关数 学知识、算法知识进行及时的预习、复习, 课下要花费一 些时间来学习, 不能光靠课上的时间, 这样可能会跟不上 老师的步伐, 课下也容易遗忘, 自己的学习可以加深印象。

\section{3. 课程的教育现状}

\section{1 教育方式}

课堂教师直接讲解各种预测方法的算法, 例如, 一次 滑动平均预测法、季节型趋势预测技术、灰色预测方法的 推导过程。老师直接向学生灌输这些知识, 而缺乏了互动 与交流, 讲义内容也比较枯燥。

考试有笔试、写论文等形式, 学生准备的程度不是很 理想。

\section{2 学生上课的积极性}

老师直接教算法的教学方式和讲义内容使得学生上课 觉得枯燥无味, 无法激起学生学习的兴趣, 学生大多没有 听课的积极性, 课下也没有进行自主学习, 上课跟不上节 奏, 因而与老师的互动也较差。另外, 考核的方式与内容 也比较容易, 学生就更没有学习该课程的积极性了。

\section{3 学习程度}

通过调查华北电力大学近几届学习过该课程的学生的 情况, 发现大多数同学对该课程的学习都是浅尝轧止, 收 获并不多。学习前没有进行数学知识的回顾, 学习过程中 觉得有些吃力, 跟不上老师的节奏, 没有听课的热情。到 了考试的时候, 也就是突击应试, 结课作业大多依靠网络 来完成, 或者是相互借鉴, 抄袭。很多都是知其然不知其 所以然。

也有极少数同学学习的效果较佳, 他们都是做了很多 努力工作的, 包括主动去学习数学知识, 课下认真做好预 习与复习, 平时与老师的交流也非常密切, 存在的问题都 通过老师、同学间的交流解决了。

整体来看, 目前的教学并没有达到理想的水平, 还需 改进, 提出教学改革势在必行。

\section{4. 调查问卷的分析}

为了调查华北电力大学关于 “电力负荷预测技术及其 应用”这门课程的实际学习情况以及同学们的意见, 制作了 调查问卷 (调查问卷见附录), 对华北电力大学学习过该课 程的同学们进行了调查, 得到了 100 份有效调查问卷。分 析调查得到的结果, 可以得出以下几点结论:

(1) 超过 50\%的同学对自己学习该课程的情况的评价 是“一般”, 很少有“好”的, 可见同学们对这门课程并没有 很大的信心;

(2) 对自己评价情况不太好的同学反映的情况大多是 数学知识与预测知识的不贯通, 上课没有兴趣, 不太听讲, 课下也没有做一些准备;

(3) 绝大多数的同学都同意在学习电力负荷预测技术 的前期应该进行一个数学相关知识的复习与整理, 他们认 为这样会对这门课程的学习有很大帮助, 因为有一些同学 就是在学习的过程中发现自己的数学知识跟不上负荷预测 的算法知识, 接着就失去了学习的积极性, 但是希望能有 老师带领着一起进行复习回顾, 课下很少有同学会主动去 做这种复习巩固工作;

（4） $67 \%$ 的同学表示同意课程中加入上机实践, 因为 老师在上课的过程中会用到一些预测的系统或软件, 但是 
仅限于在幻屏幕上展示, 同学们并没有自己跟着老师操作 的机会, 下课之后也差不多遗忘了。如果加入上机实践, 可以对电力负荷预测的算法有更加深刻的理解;

(5) 同学们对考核的方式意见是: 可以结合笔试与结 课作业一起, 这样能够培养同学们的学习能力, 也更加重 视这门课, 也可以更加全面地对学生进行考核;

(6) 同学们对该课程的一致看法是: 上课的时候不太 能跟上老师的节奏, 因为缺乏了很多相关知识, 而且课前 也没有进行预习工作, 课后也没有及时进行巩固, 没有学 习的动力与积极性;

整体上来看, 同学们还是能够认识到这门课程的重要 性的, 但是目前的教学方式让大家很少会有学习的积极性, 同学们也都很期望能够有好的教学改革, 提升同学们学习 的兴趣。

\section{5. 对课程改进提出的意见}

针对“电力负荷预测技术及其应用”这门课程的特点, 以及目前教学方式存在的问题和同学们对该问题的反馈情 况, 提出几点改进措施:

(1) 在学习电力负荷预测方法之前, 先由老师带领学 生们进行一个数学知识的复习回顾, 防止同学们在学习课 程内容时因为数学知识的遗忘而跟不上教学的节奏进而丧 失学习兴趣;

(2) 将课堂教师讲解与上机实践结合起来, 在上机课 上借助 Eviews、Matlab 等多种预测软件及工具软件加深学 生们对预测方法的理解, 提高学生们学习的兴趣, 上机课 可以布置一些上机作业, 这样也可以提高学生们的学习认 真程度和对这门课的重视程度;

(3) 采取笔试与结课作业结合的方式进行考核。笔试 的题目尽量以考核学生对算法理解性的角度去考核, 作业 以个人为单位, 并且要有一定的质量要求, 可以一定程度 上改善学生们的学习情况;

(4) 上课过程中, 增加与学生们的互动, 及时了解学 生们不懂的地方, 有针对性地进行知识的梳理和查漏补缺, 也可以提升学生们上课的积极性和集中他们的注意力。

\section{6. 结语}

电力负荷预测是电力系统规划决策、经济运行的前提 和基础, 电力负荷的准确预测对电力系统的经济运行和国 民经济的发展具有重要意义。由于电能难以大量储存的特 点, 导致了在供电过程中, 电能的生产和消费必须时刻达
到平衡。所以电力负荷的准确预测, 在国民经济, 国家发 展等问题上至关重要。而它的研究对象繁杂, 还需要进行 电力市场检测和预警。由于电力的社会属性, 还受到大量 复杂因素的多重干扰, 因此, 我们需要认真学习和研究负 荷预测理论方法, 研究如何开发实际的电力负荷预测系统, 提高负荷预测技术水平, 这样有利于提高电力系统的经济 效益和社会效益。

现在越来越多的预测方法不断涌现, 从经典的单耗法、 弹性系数法、统计分析法, 到目前的灰色预测法、专家系 统法、模糊数学法, 再到新发展的神经网络发、优选组合 法、小波分析法，以及很多新开发出来的预测软件，都对 电力负荷预测的工作带来了很大的发展和提高, 与此同时, 对负荷预测人员的技术水平要求也越来越高。而高校作为 培养人才的摇篮, 这就要求在高校内就要注意培养学生们 的能力, 就目前的教学情况来看, 课程教学改革是非常必 要的, 希望能够通过改革改善这种情况, 让大学生们在校 园里可以真正学到有用的预测方法, 对以后的工作有很大 的帮助。本文通过分析目前的教学情况以及对学生群体进 行的调查提出了几点改革建议, 希望能对教学改革有所帮 助。

\section{参考文献(References)}

[1] D.X. Niu, J.S. Lu, S.H. Cao, L. Zhao, et al. Power load forecasting technology and its application,2nd ed. China Electric Power Press, 2009.

[2] D.X. Niu, Y.L. Wang, and D. S. Dash Wu. Power load forecasting using support vector machine and ant colony optimization. Expert Systems with Applications, 37(3): 2531-2539, 2010.

[3] D.X. Niu, Y.L. Wang and X.Y. Ma. Optimization of support vector machine power load forecasting model based on data mining and Lyapunov exponents. J. Cent. South Univ. Technol, 17(2): 406-412, 2010.

[4] Y.L. Wang, D.X. Niu and X.Y. Ma. Optimizing of SVM with Hybrid PSO and Genetic Algorithm in Power Load Forecasting. Journal of Networks, 5(10): 1192-1200, 2010.

[5] Q Ding, J.G. Lu, Y.M. Qian, J. Zhang, H.Q. Liao. A practical method for ultra short term load forecasting. Automation of Electric Power Systems, 28(16): 83-85, 2004. 